\title{
Summary of papers presented at the meeting of the Society for Social Medicine in Leeds, September 1978
}

\begin{abstract}
The effectiveness of neonatal screening for congenital dislocation of the hip

D. M. PA R KIN, University of Leeds

The study looked in particular at congenital dislocation of the hip diagnosed late (after three months of age) in a cohort of births to Leeds residents in 1974-76. The incidence rate was 0.8 per 1000 births $(0.65$ and 2.23 per 1000 for hospital and GP deliveries, respectively), apparently little different from rates reported before neonatal testing was introduced. All the infants had been examined after birth. Cases detected by the family or GPs were mainly at about 12-18 months of age, with physical signs present; those by child health clinics or health visitors were found at younger ages by routine surveillance.

It was suggested that, rather than attempt to increase sensitivity of the neonatal tests (with further loss of specificity), all infants should be retested at three to six months of age, as part of the routine child health examination, looking for limited abduction of the hips.
\end{abstract}

\section{A study of acute heart attacks in Leeds}

R. D. BANDARANA Y A KE, University of Leeds A register of heart attacks occurring in the Leeds Metropolitan District was established in January 1975. Only 54\% of definite cases reached hospital. Most of the remainder were sudden deaths at home or at the place of work. The annual attack rate per 1000 population aged 40-69 was $8 \cdot 9 \%$ for males and $2 \cdot 9 \%$ for females. Major differences in length of stay, case fatality rates, and number of investigations per patient were found between the two major hospitals.

As many as $25 \%$ of all referrals to hospital were false positives. The majority of these were referred from general practice to the accident and emergency departments. Almost all had a full range of investigations before discharge.

Using a postal questionnaire on a $10 \%$ random sample of survivors two to three years after the event, it has been found that one in every six patients not retired at the time of attack were still off work. Two-thirds enjoyed life as much as they did before their illness. Almost a third said that they had not been given any information about their illness by doctors and that the advice given to patients regarding diet, smoking, exercise, occupation, and sexual activity was inadequate or totally lacking.

Delays in the diagnosis of gastro-intestinal cancer D. B. MAC ADAM, University of Leeds

The diagnoses of 150 cases of gastrointestinal cancer were inalysed retrospectively. The intervals between onset of symptoms and diagnoses by microscopy were subdivided into those occurring:

(a) between the patient first recognising that something was wrong and going to the doctor

(b) between the doctor first being told of a symptom and referring the patient for hospital investigation

(c) between referral to hospital and definitive diagnosis The cancer sites were considered in four groups: stomach, caecum and transverse colon, sigmoid colon, rectum. It was found that the interval of time at each stage of diagnosis was less than two weeks in approximately $50 \%$ of cases. In others there was delay of up to two years, so that the mean total delay between onset of symptoms and diagnosis was approximately 27 weeks. The delays occured at all three stages and were associated with the non-specific nature of many of the symptoms. Research into the symptoms of gastrointestinal cancer as they present in general practice is essential if there is to be a significant reduction in the time elapsing between the onset of symptoms and diagnosis.

The relationship between indoor air pollution from nitrogen dioxide and respiratory illness in primary schoolchildren R. J. MELIA, S. CHINN, C. DU V. FLOREY B. D. GOLDSTEIN, P. A. HUNTER, ANDE A. G. F. BROOKS, St. Thomas's Hospital Medical? School

The relationship between respiratory illness and indoor air pollution from nitrogen dioxide $\left(\mathrm{NO}_{2}\right)$ arising from gas cooking was investigated in 808 schoolchildren aged 6 and 7 living in Middlesbrough. Information on lung function, respiratory symptoms, demographic data, and kitchen $\mathrm{NO}_{2}$ measurements were available for $61 \%$ of the children. Outdoor smoke and sulphur dioxide levels were low in the area (annual averages both $<45 \mu \mathrm{g} / \mathrm{m} 3$ ). Outdoor $\mathrm{NO}_{2}$ levels measured during one week by 75 samplers ranged from 14 to $24 \mathrm{ppb}\left(10^{9}\right)$, whereas kitchen $\mathrm{NO}_{2}$ levels ranged from four to $330 \mathrm{ppb}$, and bedroom levels in 125 randomly selected homes ranged from three to $169 \mathrm{ppb}$. Kitchen levels were significantly higher in gas than in electric kitchens. The prevalence of respiratory illness was higher in children from homes with gas cookers than in those from homes with electric cookers $(\mathrm{P}<0 \cdot 10)$ after allowing for the effects of sex and social class. The number of cigarette smokers in the home did not differ between the homes with gas and those with electric cookers. The prevalence was also associated in both sexes with bedroom $\mathrm{NO}_{2}$ levels $(\mathrm{P}<0 \cdot(05)$. The distributions of social class and number of smokers did not vary with bedroom $\mathrm{NO}_{2}$ levels. As the bedroom levels were extremely low, and no association was found between the prevalence and kitchen levels, it was considered that low indoor temperatures and high humidity were likely causes of the association with gas cooking. 
Long-term consequences of respiratory disease in infancy J. M. BLAND, W. W. HOLLAND, AND

P. BAILEY, St. George's Hospital and St. Thomas's Hospital Medical Schools

It has been shown that a group of Kent children reported to have had a history of bronchitis before the age of 5 were more likely than others to be reported as having respiratory symptoms at the age of 11 . Whether the risk of reporting symptoms would change as these children grew older was considered. In four areas of Kent 1300 children were studied by parent questionnaire at the ages of 5,11, and 14 . The relative risks of reporting symptoms for children with a history of early bronchitis compared with other children were similar at the age of 14 and at the age of 11 .

In a second study of Derbyshire children aged 12, retrospective data were collected from parents about the symptoms and history of respiratory disease, and the children were asked about their current respiratory symptoms. Children whose parents reported that there was a history of bronchitis were more likely to report respiratory symptoms than children whose parents did not report such a history. This finding suggests that the prospective Kent data cannot be explained by parental bias.

Respiratory symptoms and lung function among young adults in relation to smoking and air pollution

D. IRVINE, A. G. F. BROOKS, AND

R. E. WALLER, St. Bartholomew's Hospital Medical College

A study of respiratory symptoms and ventilatory capacity among Londoners aged 18 born close to the time of the 1952 fog, was followed by a further study of a new cohort born five years later which has now been completed. Preliminary analysis of the results supports the earlier finding that smoking is the main factor affecting the prevalence of symptoms, and that respiratory illnesses in childhood affect both symptoms and ventilatory capacity. This second cohort was born at a time when smoke control was just beginning to be effective in London: comparisons between the two sets of results show little change in the overall prevalence of symptoms. A parallel sample has been studied in an unpolluted new town (Crawley). The prevalence of symptoms there is lower than in London, but lung function values are similar.

Ten-year mortality follow-up of 5000 steelworkers in relation to lung functions, respiratory symptomatology, obesity, and smoking habit

P. F. GRUNDY, Welsh National School of Medicine

In 1964 a cross-sectional study was undertaken of 80()0 steelworkers at Ebbw Vale. Data were collected on smoking habits and respiratory symptomatology, together with measurements of height, weight, and lung function. This population has now been followed for 10 years and the mortality results were presented for men who were 35 and over in 1964. Of the population of 4924 such men, details for death or survival were known for $4893(99 \cdot 4 \%)$.

The paper had two main functions: firstly, it was an attempt to clarify the relationships between the physiological measurement of lung function (in this case $\mathrm{FEV}_{1.0}$ ), recorded respiratory symptomatology, and subsequent mortality. Secondly, it provided information about the relative contributions made by smoking and obesity to total mortality and to mortality from ischaemic heart disease.

The preliminary analysis supported previous observations of the effect of smoking on mortality. In addition, the results showed that reduced $F E V_{1.0}$ was a strong predictor of death from all causes and lung cancer, and that shortness of breath predicted premature mortality from ischaemic heart disease.

Extreme overweight, expressed by a high ratio for weight $/$ height $^{2}\left(\mathrm{~W} / \mathrm{H}^{2}\right)$ predicted premature mortality in the age groups $35-44$ and $45-54$. This relationship appeared to hold for mortality from ischaemic heart disease in the younger of these two groups. The results showed that heavy smokers with a low $\mathrm{W} / \mathrm{H}^{2}$ were at higher risk of death from ischaemic heart disease than were non-smokers and ex-smokers with high $\mathrm{W} / \mathrm{H}^{2}$.

Autopsy prevalence of gallstones in nine British towns M. J. GARDNER, D. J. P. BARKER, AND C. POWE R, University of Southampton

Nine towns in England and Wales were chosen to encompass a range of socioeconomic conditions and latitude. The prevalence of gallstones in each town was measured by their frequency in all autopsies within the town from 1 January 1977 to May 1978. The survey included 5460 autopsies. The highest age- and sex-standardised prevalence, $20.6 \%$, was in Ipswich, the southern town with the best social and economic conditions. The lowest, $9 \cdot 2 \%$, was in Wakefield in the north. For women, there was a trend of increasing prevalence from north to south, in particular in towns with better social and economic conditions. There was little variation in the prevalence of solitary stones; the occurrence of multiple stones accounted for the differences between towns. On a world-wide scale, ischaemic heart disease and cholesterol gallstones, so-called diseases of civilisation, have a broadly similar distribution. However, among the nine towns, the distribution of the two disorders was strikingly different and must reflect the effect of different causal influences.

Bladder cancer in Ayrshire 1959-1972: an epidemiological and clinical study

P. BOYLE, C. R. GILLIS, W. MAC INTYR, AND J. M. GLENNIE, West of Scotland Cancer Intelligence Unit

The natural history of malignant disease of the bladder in Ayrshire has been studied epidemiologically and clinically. Both studies confirmed a significant increase in the incidence of bladder cancer in males and females which was greater than the increase for areas of the United Kingdom reported in international morbidity statistics. The increase in incidence occurred in the general population of Ayrshire and this remained true even when the known high-risk occupational groups, for example, chemical workers, farm workers, miners, and others, were omitted. This might indicate the presence of undefined population risks. This is supported by comparison of the sex ratio in other parts of 
the United Kingdom, Europe, and America, where the sex ratio is considerably higher, and by observing that the maximum age incidence in the non-occupationally related group was one decade earlier than expected.

This study has not yet revealed any clinical features, specific tumour types, or anatomical sites within the bladder which are unusual. Sixty-nine per cent of the patients presented within two months from the onset of symptoms; more than $80 \%$ of the patients presented with haematuria.

A prospective cohort study is continuing.

Clustering of places of residence of juvenile diabetics P. G. SMITH, M. THOROGOOD, AND J. I. MANN, University of Oxford

Frequent anecdotal reporting of newly diagnosed diabetic children presenting almost simultaneously from the same neighbourhood, the seasonal variation in the incidence of diabetes, and the titres of certain viral antibodies higher than expected in newly diagnosed cases, have suggested that viral infection may play a role in the aetiology of some cases of juvenile diabetes. New cases of juvenile diabetes in Oxfordshire were examined for clustering in space, or time, or space and time together, such as might be compatible with an infectious aetiology to search for evidence of a non-random distribution of cases. A significantly greater number of cases were diagnosed in the winter months September to February $(P<0 \cdot 05)$. The method of Walter was used to test for spatial clustering within districts, and significant clustering was found in one of the districts ( $P<0.001)$. Nearly all of 42 pairs of patients within the same parishes were contributed by one parish with nine patients ( 36 pairs) from a population of 4300 aged $0-16$. No evidence of 'space-time' clustering was present.

The findings are compatible with some local environmental cause, possibly a virus, but it seems unlikely that the disease is being transmitted from case to case unless the latent period of the disease is long or patients are 'infective' for a long period. A number of possible biases could have explained the findings but the statistical significance level is high, and it is suggested that similar studies be undertaken in other areas.

The relationship between alcoholic consumption and mortality from ischaemic heart disease in eighteen developed countries

A. L. COCHRANE, A. S. ST. LEGER,

F. MOORE, AND A. ZBIZOWSKI, MRC Epidemiological Research Unit, Cardiff

In a study of the factors influencing mortality in 18 developed countries, alcohol consumption (AC) was not important in spite of its unfortunate reputation. However, a strong positive correlation was found between $\mathrm{AC}$ and the death rate from liver cirrhosis, and between $\mathrm{AC}$ and carcinoma of the larynx and of the oesophagus. This suggested that AC might have some beneficial effects if it were not related to total mortality. A search of the literature suggested that ischaemic heart disease (IHD) was a possibility, and AC was found to be strongly negatively correlated to IHD death rates. The specific correlations between wine, beer, and spirits and IHD were examined; wine consumption was more strongly negatively related to IHD death rates than beer or spirits consumption.
Variations in surgical rates between nations and regions K. MC PHERSON, University of Oxford

Rates for many surgical procedures vary to a greater extent than any plausible hypothesis concerning the variations in clinical indications would predict. Nearly ten-fold variations can be observed in cholecystectomy rates between parts of the United Kingdom and Canada, for example, without any concomitant evidence about the prevalence of gall bladder disease. Many things could explain the observed variations between countries and regions and time. Existing evidence on the epidemiology of surgical rates was reviewed, and the variations in England and Wales discussed in detail. The costs of surgical uncertainty are high, for if it were known that the lower age- and sex-standardised rates for only seven discretionary procedures were adequate, the National Health Service could save perhaps $£ 50 \mathrm{~m}$ per annum. Concern in the United States of America about 'unnecessary' surgery has led to significant advances in understanding the risks, benefits, and costs of surgery, and $\vec{\nabla}$ the determinants of its variation.

Simulation models of mass screening for cervical cancer C. J. VAN OORTMARSSEN, J. D. F. 穴 HABBEMA, G. A. DE JONG, AND P. J. VAN DER MAAS,

Erasmus University

Decision-making on mass screening for cervical cancer difficult because of limited knowledge of the effect screening programmes. Evaluation of existing programme is also difficult.

In recent years, mathematical models have beêी proposed to investigate the effect of mass screening, and estimate the yield of alternative screening policies. The $\vec{\theta}$ model developed by Knox has been one of the most usefư until now. This model has been extended and applied to Dutch data on cervical cancer screening, and an estimated long-term drop in mortality of $30 \%$ was found, due to mass screening.

Measuring the quality of outpatient care: a comparison between adherence to explicit criteria and peer review I. RUSSELL, B. S. HULKA, AND N. E. CLAPP, University of North Carolina

A self-selected sample of 31 North Carolina physicians used the Delphi method to identify explicit criteria which a majority of them regarded as 'essential' in the treatment of diabetes, hypertension, and acute dysuria in females, and in general examinations. The medical records of 869 patients treated by these physicians for these conditions were scrutinised; the average percentage of consensus criteria to which physicians adhered ranged from $54 \%$ for dysuria to $69 \%$ for general examinations. One hundred and fifty-six of these records were selected at random, translated into narrative, and reviewed by a panel of 10 physicians.

Each method has its disadvantages; the measurement of explicit adherence is subject to the quality of medical $N$ records, whereas peer review is more costly and subject to more observer bias. The correlation between adherence and $N$ review scores exceeded $(\mathbf{0} \cdot \mathbf{7 0}$ for three conditions but fell to $\mathrm{N}$ ().50 for dysuria. In none of the conditions was there any association between adherence score and patient outcome. 
Manpower in the community nursing service

J. HUGHES, P. STOCKTON, J. A. ROBERTS AND R. F. L. LOGAN, London School of Hygiene and Tropical Medicine

Studies were commissioned by the North Thames Regional Health Authorities to compile basic information about manpower in the community nursing service, the development of which is essential to the implementation of the proposed change in emphasis towards community health care. Problems were found in staffing the health visiting and home nursing services in inner London, where shifting the balance of care presents the greatest difficulties.

Personnel records were the source of data on characteristics of the present stock of nurses in the community and leavers from the service. These findings highlight the complexity of the problem of achieving an adequately staffed community nursing service which provides continuity of care. Unless inner London staffing difficulties can be alleviated, it seems unlikely that community nursing teams will be able to undertake the central role in primary health care which has been envisaged for them.

\section{The work of the community physician in England} R. J. DONALDSON, AND D. J. HALL, London School of Hygiene and Tropical Medicine

A diary survey of all NHS community physicians in England, carried out over the seven-day period 5-11 December 1977 , had a response rate of $84 \%$ and produced detailed and comprehensive data about their work. The study considered the number of hours worked and the activities engaged in, by type, task, patient-client group, branch of the service involved, and underlying reason for the activity. It also examined the types of person with whom the community physician was communicating and the quality of communication.

\section{The outcome of children of low birthweight}

\section{in one regional health authority}

E. D. ALBERMAN, J. M. COLLINGWOOD, M. R. ELLIOTT, AND P.O. D. PHAROAH, Loridon School of Hygiene and Tropical Medicine

A cohort study was undertaken of all births of $2000 \mathrm{~g}$ or less in the years 1970 and 1971 to mothers resident in one regional health authority.

The proportion of survivors in three areas within the region ranged from $52 \cdot 8 \%$ to $55 \cdot 5 \%$ of all live births. The prevalence of handicap in the survey children was assessed from school medical records by comparing the proportion of low birthweight and control children of birthweight over $2000 \mathrm{~g}$ who: (1) were at special schools, (2) were on handicap registers, (3) had cerebral palsy.

Attendance at special schools was 4.6 times more common in low birthweight children (25/456) than in the total school population born in 1970 and 1971 (448/38 688).

Children of low birthweight (29/389) were 4.9 times more often on a handicap register than matched control children (6/389).

Cerebral palsy was found in $2 \cdot 2$ per cent of children of low birthweight, whereas in the general population the estimated incidence is $2 \cdot 0$ per 1000 .
Low birthweight infants remain at considerably higher risk for handicapping disorders than control children.

Infant feeding and overweight in two Oxfordshire towns M. THOROGOOD, R. CLARK, P. HARKER, AND J. I. MANN, University of Oxford

In the study of feeding and growth in the first year of life in two Oxfordshire market towns, the incidence of overweight was similar for the 'intervention' town $(12 \%)$, where a research health visitor gave intensive advice to mothers on feeding, to that for the control town (10\%). Eighteen per cent of bottle-fed infants as against $3 \%$ of breast-fed infants were found to be overweight at one year. Seventy per cent of mothers whose husbands were in non-manual jobs began breast-feeding compared with $50 \%$ of mothers whose husbands were in manual jobs, but by the time the babies were two months old this difference had disappeared and in both groups about one-third of the mothers were breast-feeding. Apparently an increase in the health visitor establishment did not affect the incidence of overweight infants, but possibly a greater emphasis on breast-feeding might reduce the incidence.

The effect of provision of school milk on the growth of schoolchildren

I. BAKER, AND P. C. ELWOOD,

MRC Epidemiological Research Unit, Cardiff

Five hundred and eighty-one schoolchildren aged 7 and 8 who came from families of four or more children were identified. The socioeconomic circumstances of these families were such that their children were more likely to receive free school meals and other welfare benefits. Only nine children came from families in Social Classes I and II and among the remaining families, $23 \%$ of fathers were unemployed. Eight per cent of families had single parents. The children were randomly allocated within their schools to a group provided with $190 \mathrm{ml}$ of milk free daily for six school terms, or to a control group. Growth in 21.5 months by height was $9.46 \mathrm{~cm}(1.68 \mathrm{~cm} \mathrm{SD})$ in the group provided with milk and $9.18 \mathrm{~cm}(1.67 \mathrm{~cm} \mathrm{SD})$ in the controls. Growth by weight in the same period was $5.25 \mathrm{~kg} \mathrm{(2.26 \textrm {kg }}$ $\mathrm{SD})$ in the milk group and $5.12 \mathrm{~kg}(1.90 \mathrm{~kg} \mathrm{SD})$ in the control group. The differences of $2.8 \mathrm{~mm}$ for height gain and $130 \mathrm{~g}$ for weight gain do not achieve statistical significance at the $5 \%$ level of probability. The results suggest that the benefit to growth of the reintroduction of free school milk for all schoolchildren aged 7-11 would be very small.

Can we predict children's smoking from their attitudes?

M. MURRAY, B. R. BEWLEY, AND

M. R. D. JOHNSON, St. Thomas's Hospital Medical School

In 1974, a five-year longitudinal study of the smoking behaviour of approximately 6000 Derbyshire schoolchildren (then aged 11-12) was begun. One of the main aims was the identification of those psychosocial characteristics which might be associated with starting to smoke.

In 1974 and 1975 each child completed an identical questionnaire which required his reaction to a series of questions about smoking and general social behaviour. 
Differences were found between the attitudes held in 1974 by those children who began smoking in 1975 and those of the group of children who did not smoke either in 1974 or in 1975. In 1974 these 'future smokers' perceived their parents as equally anti-smoking; were equally in favour of anti-smoking restrictions; had more anti-social values; had more friends who smoked and perceived greater social pressure to smoke; considered cigarette smoking more desirable; and underestimated the health hazard of smoking.

On the basis of these results, proposals were suggested which might assist efforts to persuade children of this age not to start smoking.

\section{Induced abortion in the Wessex Health Region}

J. R. ASHTON, University of Southampton

An analysis of routinely available fertility and abortion data demonstrated differences in fertility rates, the ratios of induced abortions to births, and the proportions of National Health Service operations carried out in health districts of Wessex. With few exceptions, differences in resources did not appear to be an important consideration in determining the level of NHS provision.

Patients were interviewed to compare the experiences of a group of Wessex women obtaining operations for induced abortion outside the region with those of a group obtaining a National Health Service operation within their own health district. It was found that $85 \%$ of the Wessex women who obtained an operation outside the region would have been willing to have had a local NHS operation if this had been available. This finding, and a consideration of the gestation at operation and the method of referral, indicated that a regional day care abortion service would be a practicable proposition.

Health centres: the views and experiences of patients and their doctors

R. ANDERSON AND A. CARTWRIGHT, Institute for Social Studies in Medical Care

A study in 1977 collected information from 836 people on the electoral register about their contacts and relationships with their GPs. Some limited data were also obtained from the Department of Health and Social Security about the GPs of almost all these patients. In addition, $67 \%$ of the doctors completed a postal questionnaire about their work, their practices, and their attitudes.

Twenty-two per cent of the doctors in the sample did all or part of their general practice work from a health centre. These GPs were compared with those working from their own premises.

Patients whose doctors worked from health centres were compared with the rest, looking at their experiences, their relationships with their GPs and their satisfactions and dissatisfactions with primary care. There were a number of ways in which patients whose doctors worked in a health centre were more critical of them, possibly because they knew them rather less well.

Social support and government policy on services for the elderly

S. GREEN, A. CREESE, AND J. KAUFERT St. Thomas's Hospital Medical School

Government policy on services to the elderly is to increase the proportion of the elderly cared for in the community.
This paper examined this policy in relation to informal help from friends and relatives. Drawing on the results of a pilot study of 92 people over 75, registered in a group general practice, the balance between formal and informal help over a range of activities of daily life was examined. For some activities, for example, housework and provision of meals, there was a reasonable balance between the two sources of help, but for basic ambulation and locomotion about $5 \%$ of the sample were receiving regular help from informal sources alone.

The success of policies for community care is partly dependent on informal support. This suggests that formal services should be aimed not only at the individual but also at the supporting social network. Proposals were made for additional specific functional services and for more flexible approaches to provide regular relief for informal helpers.

An ecological analysis of health differences between districts of Amsterdam

J. D. F. HABBEMA, AND

P. J. VAN DER MAAS, Erasmus University

In 1976, the Municipal Health Service of Amsterdam undertook a study on health differences between the 89 districts of Amsterdam. Five universities and municipal services participated in this study. Data collection was iv cumbersome and took two years, mainly because severą $\vec{\sigma}$ services were involved, each with its own restrictions o@ accessibility (privacy protection) and methods and purpos鸟 of registration.

Health in a district was measured by mortality, hospita admissions, and disability according to social securit standards, subdivided according to a number of medica diagnoses. The following determinants of disease were considered: population variables (density, crowding? diversity, social stratification); environmental variables (air pollution, traffic noise, housing quality); and availability of medical resources.

Major methodological problems included the interpretation of aggregate data and of dependencies between aggregate measurements, and their possible use for policy purposes. The study will be completed in the course of 1979. Provisional conclusions include the importance of 'duration of residence' of inhabitants when analysing health differences between districts, and the importance of studying younger and older age groups separately, instead of considering one overall standardised mortality ratio.

\section{Cohort follow-up using computer linkage with routinely} collected data

D. J. HOLE, J. A. CLARKE,

V. M. HAWTHORNE, AND R. MURDOCH, West of Scotland Cancer Intelligence Unit

Validation of a computerised record linkage system for matching a defined population $(n=3062)$ with two routinely collected national data sources, mortality and inpatient discharges, has been undertaken. Comparison of identifying information restricted to surname, initials, sex, and date of birth, enabled all 'probable' matches to be listed, and a clerical scrutiny of this output was also conducted.

Accuracy of the system was ascertained by examining the full death certificates and hospital records of a stratified 
random sample of the matches. Completeness was adjudged by examining the health records of a random sample of non-matches.

Identical matches referred to the same individual in $91 \%$ and $97 \%$ of cases for mortality and inpatient linkage respectively. Non-identical matches referred to the same person in $1 \%$ of cases for mortality and $9 \%$ for inpatient linkages, although this reached $67 \%$ for high probability weightings. Deaths were found in $1 \%$ and inpatient discharges in $2 \%$ of non-matches.

Sensitivity was $65 \%$ for mortality linkage and $81 \%$ for inpatient linkage with the clerical check included. Specificity was $\mathbf{9 9 . 8 \%}$ for mortality linkage and $\mathbf{9 8 . 1 \%}$ for inpatient linkage.

How visually disabled people see at home

T. R. CULLINAN, J. SILVER, L. GOULD, A ND D. IRVING, St. Bartholomew's Hospital

Medical College

A recent survey of 15000 households in England and Wales has shown a prevalence of visual diability (less than $6 / 18$ Snellen) of $520: 100000$ home-based adults (16 years + ). Eighty per cent of visually diabled people are in their retirement years and 50\% are aged 75 or over. No more than $60 \%$ have ever attended a specialist eye clinic; among those who have done so, most sight loss was caused by cataract (28\%); macular degeneration (16\%); glaucoma $(8 \%)$; and diabetic retinopathy $(6 \%)$. Forty per cent of visually disabled adults saw worse at home than in hospital; a follow-up study has now shown this to be almost entirely due to poor home lighting, for both distance and near vision. With adequate lens correction and good home lighting the prevalence of visual diability in the community would reduce to approximately $300: 100000$ adults. The use of hand held distance and reading charts by optically untrained personnel was validated.

A multicentre trial of early mobilisation after acute myocardial infarction.

R. R. WEST AND A. H. HENDERSON, Welsh National School of Medicine

During 1973-74, 35 physicians in 13 hospitals in Wales participated in a randomised trial of early mobilisation after myocardial infarction. In this multicentre trial, 747 patients with uncomplicated, proven acute myocardial infarct were randomly allocated to be mobilised on Day 5 or Day 10 . A further 293 patients with complications on Day 5 were excluded at each physician's discretion: this group included more patients with previously known high blood pressure or with arrhythmia, dyspnoea, bradycardia, tachycardia or hypertension on admisson. Morbidity was assessed after an average interval of 17 months and no significant differences were found with respect to reinfarction, severe incapacity, persistent angina, dyspnoea, or arrhythmia. Mortality was assessed by survivorship analysis until three years after infarction: there was no significant difference in mortality until 12 months but after that time patients mobilised on Day 5 showed a slightly higher mortality (Mantel Haenszel $\chi^{2}$ test $\left.P<0 \cdot 05\right)$. The apparent difference in long-term survival of patients in this trial cannot be explained by non-comparability of the two randomly allocated groups; age, sex, past history, clinical findings on admission, and treatment in hospital were very similar for those mobilised on Day 5 and Day 10.

Confusion in the use of regression and correlation analysis for the study of disease associations

A. S. ST. LEGER AND P. M. SWEETNAM, MRC Epidemiology Unit, Cardiff

Ordinary regression and correlation analyses did not give a measure of the relative specificity of association of one independent variable (for example, water calcium) to several different dependent variables (for example, various death rates). An index called the standardised regression effect (SRE) was proposed.

The SRE is the percentage change in the dependent variable consequent upon increasing the value of the given independent variable by one standard deviation from its mean value, all other independent variables in the regression set remaining fixed at their mean values.

Conditions necessary for the validity of this approach were discussed. When applicable, it makes it possible to compare the relative degree of association between one independent variable and several different dependent variables, and also to compare the relative effects of different variables on the same dependent variable. 